\title{
A Study of Weighted Polynomial Approximations with Several Variables (II)
}

\author{
Ryozi Sakai \\ Department of Mathematics, Meijo University, Tenpaku-ku, Nagoya, Japan \\ Email: ryozi@crest.ocn.ne.jp
}

How to cite this paper: Sakai, R. (2017) A Study of Weighted Polynomial Approximations with Several Variables (II). Applied Mathematics, 8, 1239-1256.

https://doi.org/10.4236/am.2017.89093

Received: August 13, 2017

Accepted: September 3, 2017

Published: September 6, 2017

Copyright $\odot 2017$ by author and Scientific Research Publishing Inc. This work is licensed under the Creative Commons Attribution International License (CC BY 4.0).

http://creativecommons.org/licenses/by/4.0/

\begin{abstract}
In this paper we investigate weighted polynomial approximations with several variables. Our study relates to the approximation for $W f \in L^{p}\left(\mathbb{R}^{s}\right)$ by weighted polynomial. Then we will give some results relating to the Lagrange interpolation, the best approximation, the Markov-Bernstein inequality and the Nikolskii-type inequality.
\end{abstract}

\section{Keywords}

Weighted Polynomial Approximations, the Lagrange Interpolation, the Best of Approximation, Inequalities

\section{Introduction}

Let $\mathbb{R}^{s}:=\mathbb{R} \times \mathbb{R} \times \cdots \times \mathbb{R}$ ( $s$ times, $s \geq 1$ integer) be the direct product space, and let $W\left(x_{1}, x_{2}, \cdots, x_{s}\right):=w_{1}\left(x_{1}\right) w_{2}\left(x_{2}\right) \cdots w_{s}\left(x_{s}\right)$, where $w_{i}\left(x_{i}\right) \geq 0$ be even weight functions. We suppose that for every nonnegative integer $n$,

$$
\int_{0}^{\infty} x^{n} w_{i}(x) \mathrm{d} x<\infty, n=0,1,2, \cdots, i=1,2, \cdots, s .
$$

In this paper we will study to approximate the real-valued weighted function (Wf $)\left(x_{1}, x_{2}, \cdots, x_{s}\right)$ by weighted polynomials $(W P)\left(x_{1}, x_{2}, \cdots, x_{s}\right)$, where $P\left(x_{1}, x_{2}, \cdots, x_{s}\right) \in \mathcal{P}_{n, n, \cdots, n}\left(\mathbb{R}^{s}\right)$. Here, $\mathcal{P}_{n, n, \cdots, n}\left(\mathbb{R}^{s}\right)\left(=: \mathcal{P}_{n ; s}\left(\mathbb{R}^{s}\right)\right)$ means a class of all polynomials with at most $n$-degree for each variable $x_{i}, i=1,2, \cdots, s$. We need to define the norms. Let $0<p \leq \infty$, and let $f: \mathbb{R}^{s} \rightarrow \mathbb{R}$ be measurable. Then we define

$$
\|W f\|_{L^{p}\left(\mathbb{R}^{s}\right)}:= \begin{cases}{\left[\int_{-\infty}^{\infty} \cdots \int_{-\infty}^{\infty}\left|(W f)\left(x_{1}, \cdots, x_{s}\right)\right|^{p} \mathrm{~d} x_{1} \cdots \mathrm{d} x_{s}\right]^{1 / p},} & \text { if } 0<p<\infty ; \\ \sup _{\left(x_{1}, \cdots, x_{s}\right) \in \mathbb{R}^{s}}\left|(W f)\left(x_{1}, \cdots, x_{s}\right)\right|, & \text { if } p=\infty .\end{cases}
$$


We assume that for $0<p \leq \infty$ the integral is independent of the order of integration with respect to each $x_{i}, i=1,2, \cdots, s$. When $\|W f\|_{L^{p}\left(\mathbb{R}^{s}\right)}<\infty$, we write $W f \in L^{p}\left(\mathbb{R}^{s}\right)$. If $p=\infty$, we require that $f$ is continuous and

$\lim _{|X| \rightarrow \infty} W(X) f(X)=0$, where $|X|=\left|\left(x_{1}, \cdots, x_{s}\right)\right|=\max \left|x_{i}\right| ; i=1,2, \cdots, s$. Then we write $W f \in C_{0}\left(\mathbb{R}^{s}\right)$.

Our purpose in this paper is to approximate the weighted function $W f \in L^{p}\left(\mathbb{R}^{s}\right)$ by weighted polynomials $W P ; P \in \mathcal{P}_{n ; s}\left(\mathbb{R}^{s}\right)$. In Section 2, we give a class of the weights which are treated in this paper. In Section 3, we state our main theorems. First, we consider the Lagrange interpolation polynomials. Next, we give the necessary and sufficient conditions for the best approximation. In Sections 4 and 5, we will prove theorems.

\section{Class of Weight Functions and Preliminaries}

Throughout the paper $C, C_{1}, C_{2}, \cdots$ denote positive constants independent of $n, x, t$ or polynomials $P(x)$. The same symbol does not necessarily denote the same constant in different occurrences. Let $f(x) \sim g(x)$ mean that there exists a constant $C>0$ such that $C^{-1} f(x) \leq g(x) \leq C f(x)$ holds for all $x \in I$, where $I \subset \mathbb{R}$ is a subset.

We say that $f: \mathbb{R} \rightarrow[0, \infty)$ is quasi-increasing if there exists $C>0$ such that $f(x) \leq C f(y)$ for $0<x<y$. Hereafter we consider following weights.

Definition 2.1. Let $Q: \mathbb{R} \rightarrow[0, \infty)$ be a continuous and even function, and satisfy the following properties:

(a) $Q^{\prime}(x)$ is continuous in $\mathbb{R}$, with $Q(0)=0$.

(b) $Q^{\prime \prime}(x)$ exists and is positive in $\mathbb{R} \backslash\{0\}$.

(c) $\lim _{x \rightarrow \infty} Q(x)=\infty$.

(d) The function

$$
T(x):=\frac{x Q^{\prime}(x)}{Q(x)}, x \neq 0
$$

is quasi-increasing in $(0, \infty)$, with

$$
T(x) \geq \Lambda>1, x \in \mathbb{R} \backslash\{0\} .
$$

(e) There exists $C_{1}>0$ such that

$$
\frac{Q^{\prime \prime}(x)}{\left|Q^{\prime}(x)\right|} \leq C_{1} \frac{\left|Q^{\prime}(x)\right|}{Q(x)} \text {, a.e. } x \in \mathbb{R} \text {. }
$$

Then we write $w=\exp (-Q) \in \mathcal{F}\left(C^{2}\right)$.

Moreover, if there also exists a compact subinterval $J(\ni 0)$ of $\mathbb{R}$, and $C_{2}>0$ such that

$$
\frac{Q^{\prime \prime}(x)}{\left|Q^{\prime}(x)\right|} \geq C_{2} \frac{\left|Q^{\prime}(x)\right|}{Q(x)} \text {, a.e. } x \in \mathbb{R} \backslash J,
$$

then we write $w=\exp (-Q) \in \mathcal{F}\left(C^{2}+\right)$. If $T(x)$ is bounded, then the weight 
$w=\exp (-Q) \in \mathcal{F}\left(C^{2}+\right)$ is called a Freud-type weight, and if $T(x)$ is unbounded, then $w$ is called an Erdös-type weight.

Let $w(x)=\exp (-Q(x)) \in \mathcal{F}\left(C^{2}+\right), \quad 0<\lambda<(m+2) /(m+1)$ and $m \geq 1$ be an integer. Then we write $w \in \mathcal{F}_{\lambda}\left(C^{m+2}+\right)$ if $Q$ is $C^{m+2}$-class and there exist $C \geq 1$ and $K \geq 1$ such that for all $|x| \geq K$,

$$
\frac{\mid Q^{\prime}(x)}{Q(x)^{\lambda}} \leq C
$$

and

$$
\left|\frac{Q^{\prime \prime}(x)}{Q^{\prime}(x)}\right| \sim\left|\frac{Q^{(k+1)}(x)}{Q^{(k)}(x)}\right|
$$

for every $k=2, \cdots, m$ and also

$$
\left|\frac{Q^{(m+2)}(x)}{Q^{(m+1)}(x)}\right| \leq\left|\frac{Q^{(m+1)}(x)}{Q^{(m)}(x)}\right| .
$$

Specific examples are shown in the following:

Example 2.2 (cf. [1] [2]). (1) If an exponential $Q(x)$ satisfies

$$
1<\Lambda_{1} \leq \frac{\left(x Q^{\prime}(x)\right)^{\prime}}{Q^{\prime}(x)} \leq \Lambda_{2},
$$

where $\Lambda_{i}, i=1,2$ are constants, then we call $w=\exp (-Q(x))$ the Freud weight. The class $\mathcal{F}\left(C^{2}+\right)$ contains the Freud weights.

(2) For $\alpha>1, l \geq 1$ we define

$$
Q(x)=Q_{l ; \alpha}(x)=\exp _{l}\left(|x|^{\alpha}\right)-\exp _{l}(0),
$$

where $\exp _{l}(x)=\exp (\exp (\exp \cdots \exp x) \cdots)(l$ times $)$. Moreover, we define

$$
Q_{l ; \alpha, m}(x)=|x|^{m}\left\{\exp _{l}\left(|x|^{\alpha}\right)-\alpha^{*} \exp _{l}(0)\right\}, \alpha+m>1, m \geq 0, \alpha \geq 0,
$$

where $\alpha^{*}=0$ if $\alpha=0$, and otherwise $\alpha^{*}=1$. We note that $Q_{1 ; 0, m}$ gives a Freud-type weight, that is, $T(x)$ is bounded..

(3) We define

$$
Q_{\alpha}(x)=(1+|x|)^{|x|^{\alpha}}-1, \alpha>1 .
$$

(4) Let $w=\exp (-Q) \in \mathcal{F}\left(C^{2}+\right)$, and let us define

$$
\mu_{+}:=\limsup _{x \rightarrow \infty} \frac{Q^{\prime \prime}(x)}{Q^{\prime}(x)} / \frac{Q^{\prime}(x)}{Q(x)}, \mu_{-}:=\liminf _{x \rightarrow \infty} \frac{Q^{\prime \prime}(x)}{Q^{\prime}(x)} / \frac{Q^{\prime}(x)}{Q(x)} .
$$

If $\mu_{+}=\mu_{-}$, then we say that the weight $w$ is regular. All weights in examples (1), (2) and (3) are regular.

(5) More generally we can give the examples of weights $w \in \mathcal{F}_{\lambda}\left(C^{m+2}+\right)$. If 
the weight $w$ is regular and if $Q \in \mathbf{C}^{m+2}(\mathbb{R} \backslash\{0\})$ satisfies definition (2.1), then for the regular weights we have $w \in \mathcal{F}_{\lambda}\left(C^{m+2}+\right)$ (see [3], Corollary 5.5 (5.8)).

Proposition 2.3 ([3], Theorem 4.2 and (4.11)). Let $m$ be a positive integer, $0<\lambda<(m+2) /(m+1)$ and let $w=\exp (-Q) \in \mathcal{F}_{\lambda}\left(C^{m+2}+\right)$. Then for $\mu, \nu, \alpha, \beta \in \mathbb{R}$, we can construct a new weight $w_{\mu, v, \alpha, \beta} \in \mathcal{F}_{\lambda}\left(C^{m+1}+\right) \subset \mathcal{F}\left(C^{2}+\right)$ such that

$$
T_{w}(x)^{\mu}\left(1+x^{2}\right)^{v}(1+Q(x))^{\alpha}\left(1+\left|Q^{\prime}(x)\right|\right)^{\beta} w(x) \sim w_{\mu, v, \alpha, \beta}(x) \text { on } \mathbb{R},
$$

and for some $C \geq 1$,

$$
a_{n / C}\left(w_{\mu, v, \alpha, \beta}\right) \leq a_{n}(w) \leq a_{C n}\left(w_{\mu, v, \alpha, \beta}\right) \text { and } T_{w_{\mu, v, \alpha, \beta}}(x) \sim T_{w}(x)=T(x),
$$

where $a_{n}\left(w_{\mu, v, \alpha, \beta}\right)$ and $a_{n}(w)$ are MRS-numbers for the weight $w_{\mu, \nu, \alpha, \beta}$ or $w$, respectively, and $T_{w_{\mu, \nu, \alpha, \beta}}, T_{w}$ are correspond for $w_{\mu, \nu, \alpha, \beta}$ or $w$, respectively.

Let $\left\{p_{n}\right\}$ be orthonormal polynomials with respect to a weight $w$, that is, $p_{n}$ is the polynomial of degree $n$ such that

$$
\int_{-\infty}^{\infty} p_{n}(x) p_{m}(x) w^{2}(x) \mathrm{d} x=\delta_{m n} \text { (the Kronecker delta). }
$$

For $1 \leq p \leq \infty$, we denote by $L^{p}(\mathbb{R})$ the usual $L^{p}$ space on $\mathbb{R}$ (here for $p=\infty$, if $w f \in L^{\infty}(\mathbb{R})$ then we require $f$ to be continuous, and $f w$ to have limit 0 at $\pm \infty)$. Let $w \in \mathcal{F}\left(C^{2}+\right)$. We need the Mhaskar-Rakhmanov-Saff numbers (MRS numbers) $a_{x}$;

$$
x=\frac{2}{\pi} \int_{0}^{1} \frac{a_{x} u Q^{\prime}\left(a_{x} u\right)}{\left(1-u^{2}\right)^{1 / 2}} \mathrm{~d} u, x>0 .
$$

we see easily

$$
\lim _{x \rightarrow \infty} a_{x}=\infty \text { and } \lim _{x \rightarrow+0} a_{x}=0
$$

and

$$
\lim _{x \rightarrow \infty} \frac{a_{x}}{x}=0 \text { and } \lim _{x \rightarrow+0} \frac{a_{x}}{x}=\infty .
$$

For $w f \in L_{p}(\mathbb{R})(1 \leq p \leq \infty)$ the degree of weighted polynomial approximation is defined by

$$
E_{n, p}(w ; f):=\inf _{P \in \mathcal{P}_{n}}\|w(f-P)\|_{L^{p}(\mathbb{R})} .
$$

\section{Main Results}

Let $w_{i} \in \mathcal{F}\left(C^{2}+\right), i=1,2, \cdots, s$, and let $W(X)=\prod_{i=1}^{s} w_{i}\left(x_{i}\right)$, where $X=\left(x_{1}, x_{2}, \cdots, x_{s}\right) \in \mathbb{R}^{s}$. Then we have the following theorem.

Theorem 3.1 ([4], Theorem 3.3). We suppose

$$
\begin{gathered}
w_{j}=\exp \left(-Q_{j}\right) \in \mathcal{F}_{\lambda}\left(C^{3}+\right)(0<\lambda<3 / 2), \quad j=1,2, \cdots, s \text { and let } \\
T_{j}\left(a_{n}^{(j)}\right) \leq c\left(\frac{n}{a_{n}^{(j)}}\right)^{2 / 3}, j=1,2, \cdots, s .
\end{gathered}
$$


If $\prod_{i=1}^{s}\left\{T_{i}^{1 / 4} w_{i}\right\} f \in C_{0}\left(\mathbb{R}^{s}\right)$, then there exist $P_{n} \in \mathcal{P}_{n ; s}\left(\mathbb{R}^{s}\right), n=1,2,3, \cdots$ such that we have

$$
\left\|W\left(f-P_{n}\right)\right\|_{L^{\infty}\left(\mathbb{R}^{s}\right)} \rightarrow 0 \text { as } n \rightarrow \infty .
$$

First, we consider the Lagrange interpolation operators. We construct the orthonormal polynomials $p_{n, i}$ with respect to the weight $w_{i}$ for each $i=1,2, \cdots, s$. Let $x_{n, n, i}<x_{n-1, n, i}<\cdots<x_{1, n, i}, i=1,2, \cdots, s$ are zeros of the orthonormal polynomial $p_{n, i}$, that is, $p_{n, i}\left(x_{k_{i}, n, i}\right)=0, k_{i}=1,2, \cdots, n$ and put $S_{n}=\left\{\left(x_{k_{1}, n, 1}, \cdots, x_{k_{s}, n, s}\right) ; 1 \leq k_{i} \leq n, i=1,2, \cdots, s\right\}$. Then for $W f \in C_{0}(\mathbb{R})$ we define the Lagrange interpolation polynomial on $S_{n}$ as

$$
L_{n}\left(f ; x_{1}, \cdots, x_{s}\right)=\sum_{k_{s}=1}^{n} \cdots \sum_{k_{1}=1}^{n} f\left(x_{k_{1}, n, 1}, \cdots, x_{k_{s}, n, s}\right) \prod_{i=1}^{s} l_{k_{i}, n, i}\left(x_{i}\right),
$$

where

$$
l_{k_{i}, n, i}\left(x_{i}\right)=\frac{p_{n, i}\left(x_{i}\right)}{\left(x_{i}-x_{k_{i}, n, i}\right) p_{n, i}^{\prime}\left(x_{k_{i}, n, i}\right)} .
$$

In the rest of this paper, if $w_{i}, i=1,2, \cdots, s$ are the Freud-type weights then we suppose $a_{n}^{(i)}=o(1) n^{2 / 3}$.

Theorem 3.2. Let $w_{i} \in \mathcal{F}\left(C^{2}+\right), i=1,2, \cdots, s$, and let $f: \mathbb{R}^{s} \rightarrow \mathbb{R}$ be continuous. If

$$
\prod_{i=1}^{s}\left\{\left(1+x_{i}^{2}\right)^{\beta / 2} w_{i}\left(x_{i}\right)\right\}\left|f\left(x_{1}, \cdots, x_{s}\right)\right| \in C_{0}\left(\mathbb{R}^{s}\right)
$$

holds, then there exists $n_{0}>0$ such that for $n \geq n_{0}$

$$
\begin{aligned}
& \left|\sum_{k_{s}=1}^{n} \cdots \sum_{k_{1}=1}^{n} \prod_{i=1}^{s} \lambda_{k_{i}, n, i} f\left(x_{k_{1}, n, 1}, \cdots, x_{k_{s}, n, s}\right)\right| \\
& \leq C\left\|\prod_{i=1}^{s}\left\{\left(1+x_{i}^{2}\right)^{\beta} w_{i}^{2}\left(x_{i}\right)\right\} f\left(x_{1}, \cdots, x_{s}\right)\right\|_{L^{\infty}\left(\mathbb{R}^{s}\right)},
\end{aligned}
$$

where for each $i=1,2, \cdots, s, \quad x_{k_{i}, n, i}\left(k_{i}=1,2, \cdots, n\right)$ are zeros of $p_{n, i}\left(x_{i}\right)$. In particular,

$$
\begin{aligned}
& \lim _{n \rightarrow \infty} \sum_{k_{s}=1}^{n} \cdots \sum_{k_{1}=1}^{n} \prod_{i=1}^{s} \lambda_{k_{i}, n, i} f\left(x_{k_{1}, n, 1}, \cdots, x_{k_{s}, n, s}\right) \\
& =\int_{-\infty}^{\infty} \cdots \int_{-\infty}^{\infty} \prod_{i=1}^{s} w_{i}^{2}\left(x_{i}\right) f\left(x_{1}, \cdots, x_{s}\right) \mathrm{d} x_{1} \cdots \mathrm{d} x_{s} .
\end{aligned}
$$

Theorem 3.3. Let $w_{i} \in \mathcal{F}_{\lambda}\left(C^{3}+\right)(0<\lambda<3 / 2), i=1,2,3, \cdots, s$. Let $\beta>1 / 2$, and let $f: \mathbb{R}^{s} \rightarrow \mathbb{R}$ satisfy (3.3), then we have for $n=1,2, \cdots$,

$$
\left\|\prod_{i=1}^{s} w_{i} L_{n}(f)\right\|_{L^{\infty}\left(\mathbb{R}^{s}\right)} \leq C\left\|\prod_{i=1}^{s}\left\{\left(1+x_{i}^{2}\right)^{(\beta / 2)} w_{i}\left(x_{i}\right)\right\} f\left(x_{1}, \cdots, x_{s}\right)\right\|_{L^{\infty}\left(\mathbb{R}^{s}\right)},
$$

where for each $i=1,2, \cdots, s, \quad x_{k_{i}, n, i}, k_{i}=1,2, \cdots, n$ are zeros of $p_{n, i}\left(x_{i}\right)$. In particular, if $\prod_{i=1}^{s}\left\{\left(1+x_{i}^{2}\right)^{(\beta / 2)} T_{i}^{1 / 4}\left(x_{i}\right) w_{i}\left(x_{i}\right)\right\} f\left(x_{1}, \cdots, x_{s}\right) \in C_{0}\left(\mathbb{R}^{s}\right)$, then we 
have

$$
\lim _{n \rightarrow \infty}\left\|\prod_{i=1}^{s} w_{i}\left(f-L_{n}(f)\right)\right\|_{L^{2}\left(\mathbb{R}^{s}\right)}=0
$$

For $p \neq 2$ we also obtain the similar results. We need a function as follows:

$$
\Psi(x):=\frac{1}{(1+Q(x))^{2 / 3} T(x)} .
$$

Theorem 3.4. Let $w_{i} \in \mathcal{F}_{\lambda}\left(C^{3}+\right), 0<\lambda<3 / 2(i=1,2, \cdots, s)$. Let $1<p \leq 2$ and $\beta>1 / p$, and let $\Psi(x)$ be defined by (3.7) for each $w_{i}, i=1,2, \cdots, s$. If $f: \mathbb{R} \rightarrow \mathbb{R}$ is continuous, and satisfies

$$
\begin{aligned}
& \left|\prod_{i=1}^{s}\left\{\left(1+x_{i}^{2}\right)^{\beta / 2} T_{i}^{1 / 2}\left(x_{i}\right) \Psi_{i}^{-1 / 4}\left(x_{i}\right)\right\} W(X) f(X)\right|<\infty, \\
& X \in \mathbb{R}^{s}, i=1,2, \cdots, s,
\end{aligned}
$$

then we have

$$
\begin{aligned}
& \left\|W L_{n}(f)\right\|_{L^{p}\left(\mathbb{R}^{s}\right)} \leq C\left\|\prod_{i=1}^{s}\left\{\left(1+x_{i}^{2}\right)^{\beta / 2} T_{i}^{1 / 2}\left(x_{i}\right) \Psi_{i}^{-1 / 4}\left(x_{i}\right)\right\} W f\right\|_{L^{\infty}\left(\mathbb{R}^{s}\right)}, \\
& n=1,2, \cdots
\end{aligned}
$$

Especially, if $f$ satisfies

$$
\left|\prod_{i=1}^{s}\left\{\left(1+x_{i}^{2}\right)^{\beta / 2} T_{i}^{3 / 4}\left(x_{i}\right) \Psi_{i}^{-1 / 4}\left(x_{i}\right)\right\} W(X) f(X)\right| \in C_{o}\left(\mathbb{R}^{s}\right),
$$

then we have

$$
\lim _{n \rightarrow \infty}\left\|W\left(f-L_{n}(f)\right)\right\|_{L^{p}\left(\mathbb{R}^{s}\right)}=0 .
$$

For $2<p \leq \infty$ we have the following:

Theorem 3.5. Let $w_{i} \in \mathcal{F}_{\lambda}\left(C^{3}+\right), 0<\lambda<3 / 2(i=1,2, \cdots, s)$, and let satisfy $T_{i}\left(a_{n}^{(i)}\right) \leq C n^{1 / 2}$. Let $2<p \leq \infty$ and $\beta>1 / p$. Furthermore we assume

$$
Q_{i}\left(\frac{a_{n}^{(i)}}{4}\right) \geq C\left(\log \left(Q_{i}\left(a_{n}^{(i)}\right)\right)\right)^{4}, i=1,2, \cdots, s .
$$

If $f: \mathbb{R}^{s} \rightarrow \mathbb{R}$ is continuous, and satisfies

$$
\left|\prod_{i=1}^{s}\left\{\left(1+x_{i}^{2}\right)^{\beta / 2} T_{i}^{1 / 2}\left(x_{i}\right) \Psi_{i}^{-1}\left(x_{i}\right)\right\} W(X) f(X)\right|<\infty, X \in \mathbb{R}^{s},
$$

then we have

$$
\begin{aligned}
& \left\|\left\{\prod_{i=1}^{s} \Psi_{i}^{3 / 4}\left(x_{i}\right)\right\} W L_{n}(f)\right\|_{L^{p}\left(\mathbb{R}^{s}\right)} \\
& \leq C\left\|\prod_{i=1}^{s}\left\{\left(1+x_{i}^{2}\right)^{\beta / 2} T_{i}^{1 / 2}\left(x_{i}\right) \Psi_{i}^{-1 / 4}\left(x_{i}\right)\right\} W f\right\|_{L^{\infty}\left(\mathbb{R}^{s}\right)} .
\end{aligned}
$$

Especially, by (3.13) we have 


$$
\lim _{n \rightarrow \infty}\left\|\left\{\prod_{i=1}^{s} \Psi_{i}^{3 / 4}\left(x_{i}\right)\right\} W\left(f-L_{n}(f)\right)\right\|_{L^{p}\left(\mathbb{R}^{s}\right)}=0
$$

Remark 3.6. (1) We note that (3.13) means

$$
\left(\prod_{i=1}^{s} T_{i}^{1 / 4}\right) W^{*} f \in C_{0}\left(\mathbb{R}^{s}\right)
$$

where $\prod_{i=1}^{s}\left\{\left(1+x_{i}^{2}\right)^{\beta / 2} T_{i}^{1 / 2}\left(x_{i}\right) \Psi_{i}^{-1 / 4}\left(x_{i}\right)\right\} W \sim W^{*} \in \mathcal{F}\left(C^{2}+\right) \quad$ (see $\quad$ Theorem 2.3).

(2) All examples in Example 2.2 hold (3.12).

(3) To prove Theorem 3.5 we use Proposition 4.5. Then Assumption (3.12) plays an important role.

Next, we characterize the best approximation polynomial (cf. [5]).

Theorem 3.7. Let $0<p \leq \infty$. There is a best approximation polynomial $P_{f} \in \mathcal{P}_{n ; s}$ such that

$$
E_{p, n ; s}(W, f):=\inf _{P \in \mathcal{P}_{n ; s}}\|W(f-P)\|_{L^{p}\left(\mathbb{R}^{s}\right)}=\left\|W\left(f-P_{n ; f}\right)\right\|_{L^{p}\left(\mathbb{R}^{s}\right)} .
$$

Theorem 3.8 (Kolmogorov-type theorem). $P \in \mathcal{P}_{n ; s}$ is a best of approximation for a continuous function $f$ with $\lim _{\left|\left(x_{1}, \cdots, x_{s}\right)\right| \rightarrow \infty} W\left(x_{1}, \cdots, x_{s}\right) f\left(x_{1}, \cdots, x_{s}\right)=0$, if and only if for each polynomial $Q \in \mathcal{P}_{n ; s}$,

$$
\max _{\left(x_{1}, \cdots, x_{s}\right) \in A}\left[W\left(x_{1}, \cdots, x_{s}\right)\left(f\left(x_{1}, \cdots, x_{s}\right)-P\left(x_{1}, \cdots, x_{s}\right)\right)\right] Q\left(x_{1}, \cdots, x_{s}\right) \geq 0,
$$

where $A$ denotes the set (which depends on $f$ and $P$ ) of all points $\left(x_{1}, \cdots, x_{s}\right) \in A$ for which

$$
\left|W\left(x_{1}, \cdots, x_{s}\right)\left(f\left(x_{1}, \cdots, x_{s}\right)-P\left(x_{1}, \cdots, x_{s}\right)\right)\right|=\|W(f-P)\|_{L^{\infty}\left(\mathbb{R}^{s}\right)} .
$$

Theorem 3.9. Let $W=\prod_{i=1}^{s} w_{i}, w_{i} \in \mathcal{F}\left(C^{2}+\right), i=1, \cdots, s$ and $1 \leq p<\infty$. Let $\phi_{K}$, where $K=\left(k_{1}, \cdots, k_{s}\right)\left(0 \leq k_{j} \leq n\right)(j=1, \cdots, s)$ be a linearly independent system satisfying $W \phi_{K} \in L_{p}\left(\mathbb{R}^{s}\right)$, and we consider polynomials

$$
Q(X)=\sum_{k_{s}=1}^{n} \cdots \sum_{k_{1}=1}^{n} c_{\left(k_{1}, \cdots, k_{s}\right)} \phi_{\left(k_{1}, \cdots, k_{s}\right)}(X) .
$$

Let $W f \in L_{p}\left(\mathbb{R}^{s}\right)$. The polynomial

$$
P(X)=\sum_{k_{s}=1}^{n} \cdots \sum_{k_{1}=1}^{n} c_{\left(k_{1}, \cdots, k_{s}\right)}^{[0]} \phi_{\left(k_{1}, \cdots, k_{s}\right)}(X)
$$

is a polynomial of the best approximation for $f$ if and only if for every polynomial (3.15) the following equality (3.17) holds.

$$
\begin{aligned}
& \int_{\mathbb{R}^{s}} Q(X)|f(X)-P(X)|^{p-1}[\operatorname{sign}(f(X)-P(X))] W^{p}(X) D X \\
& :=\int_{\mathbb{R}} \cdots \int_{\mathbb{R}} Q\left(x_{1}, \cdots, x_{s}\right)\left|f\left(x_{1}, \cdots, x_{s}\right)-P\left(x_{1}, \cdots, x_{s}\right)\right|^{p-1} \\
& \quad \times\left[\operatorname{sign}\left(f\left(x_{1}, \cdots, x_{s}\right)-P\left(x_{1}, \cdots, x_{s}\right)\right)\right] W^{p}\left(x_{1}, \cdots, x_{s}\right) \mathrm{d} x_{1} \cdots \mathrm{d} x_{s}=0
\end{aligned}
$$


holds. If $p=1$, we also assume that $f(X)-P(X)$ vanish only on a set of measure zero.

\section{Proofs of Theorems 3.2, 3.3, 3.4 and 3.5}

Lemma 4.1. Let $x_{n, n, i}<x_{n-1, n, i}<\cdots<x_{1, n, i}$ be zeros of the orthonormal polynomial $p_{n, i}$, and let

$$
P_{n-1}\left(x_{1}, x_{2}, \cdots, x_{s}\right)=\sum_{0 \leq j_{i} \leq n-1, i=1,2, \cdots, s} a_{j_{1}, \cdots, j_{s}} x_{1}^{j_{1}} \cdots x_{s}^{j_{s}},
$$

where $a_{j_{1}, \cdots, j_{s}}$ are coefficients. If for every $\left(x_{k_{1}, n, 1}, \cdots, x_{k_{s}, n, s}\right)$,

$$
P_{n-1}\left(x_{k_{1}, n, 1}, \cdots, x_{k_{s}, n, s}\right)=0,1 \leq k_{i} \leq n, i=1, \cdots, s,
$$

then we have $P_{n-1}=0$. Therefore, for $P_{n-1}\left(x_{1}, x_{2}, \cdots, x_{s}\right) \in \mathcal{P}_{n-1}\left(\mathbb{R}^{s}\right)$ we have

$$
P_{n-1}=L_{n}\left(P_{n-1}\right) \text {. }
$$

Proof. Now we fix any $\left(x_{k_{2}, n, 2}, \cdots, x_{k_{s}, n, s}\right) \in \mathbb{R}^{s-1}$, and then we consider the polynomial $Q_{n-1}\left(x_{1}\right)$ in $\mathcal{P}_{n-1}(\mathbb{R})$ such that

$$
Q_{n-1}\left(x_{1}\right)=\sum_{j_{1}=0}^{n-1}\left(\sum_{0 \leq j_{i} \leq n-1, i=2,3, \cdots, s} a_{j_{1}, \cdots, j_{s}} x_{k_{2}, n, 2}^{j_{2}} \cdots x_{k_{s}, n, s}^{j_{s}}\right) x_{1}^{j_{1}} .
$$

Since

$$
Q_{n-1}\left(x_{k_{1}, n, 1}\right)=0, k_{1}=1,2, \cdots, n
$$

(see (4.1)), all coefficients of $Q_{n-1}\left(x_{1}\right)$ equal to zero, that is,

$$
\sum_{0 \leq j_{i} \leq n-1, i=2,3, \cdots, s} a_{j_{1}, \cdots, j_{s}} x_{k_{2}, n, 2}^{j_{2}} \cdots x_{k_{s}, n, s}^{j_{s}}=0 \text {, for each } j_{1}=0,1, \cdots, n-1 \text {. }
$$

Next, we fix any

$$
j_{1}\left(0 \leq j_{1} \leq n-1\right) \text { and }\left(x_{k_{3}, n, 3}, \cdots, x_{k_{s}, n, s}\right) \in \mathbb{R}^{s-2},
$$

and we consider $R_{n-1} \in \mathcal{P}_{n-1}(\mathbb{R})$ such that

$$
R_{n-1}\left(x_{2}\right)=\sum_{j_{2}=0}^{n-1}\left(\sum_{0 \leq j_{i} \leq n-1, i=3,4, \cdots, s} a_{j_{1}, \cdots, j_{s}} x_{k_{3}, n, 3}^{j_{3}} \cdots x_{k_{s}, n, s}^{j_{s}}\right) x_{2}^{j_{2}} .
$$

Then by (4.3), we see

$$
R_{n-1}\left(x_{k_{2}, n, 2}\right)=0, k_{2}=1,2, \cdots, n .
$$

Hence,

$$
\sum_{0 \leq j_{i} \leq n-1, i=3,4, \cdots, s} a_{j_{1}, \cdots, j_{s}} x_{3, k_{3}}^{j_{3}} \cdots X_{s, k_{s}}^{j_{s}}=0 \text {, for each } j_{1}, j_{2}=0,1, \cdots, n-1 \text {. }
$$

If we continue this method inductively, then we have

$$
\sum_{j_{s}=0}^{n-1} a_{j_{1}, \cdots, j_{s}} x_{k_{s}, n, s}^{j_{s}}=0 \text {, for each } j_{1}, j_{2}, \cdots, j_{s-1}=0,1, \cdots, n-1 \text {. }
$$


We put $H_{n-1} \in \mathcal{P}_{n-1}(\mathbb{R})$ as

$$
H_{n-1}\left(x_{s}\right)=\sum_{j_{s}=0}^{n-1} a_{j_{1}, \cdots, j_{s}} x_{s}^{j_{s}},
$$

then from (4.4) we have $H_{n-1}\left(x_{k_{s}, n, s}\right)=0, k_{s}=1,2, \cdots, n$. Therefore, we conclude

$$
a_{j_{1}, \cdots, j_{s}}=0, j_{i}=0,1, \cdots, n-1(i=1,2, \cdots, s),
$$

that is, $P_{n-1}=0$.

In the rest of this paper, we use the following notations:

$$
\begin{gathered}
W=\prod_{i=1}^{s} w_{i}, X=\left(x_{1}, \cdots, x_{s}\right), X(u)=\left(u_{1}, \cdots, u_{s}\right) \\
D(X)=\mathrm{d} x_{1} \cdots \mathrm{d} x_{s}, D(X(u))=\mathrm{d} u_{1} \cdots \mathrm{d} u_{s} .
\end{gathered}
$$

We also use

$$
\begin{aligned}
\mathcal{P}_{n ; s}\left(\mathbb{R}^{s}\right):=\mathcal{P}_{n, \cdots, n}\left(\mathbb{R}^{s}\right):= & \left\{P_{n} \mid P_{n}\left(x_{1}, \cdots, x_{s}\right) \text { are polynomials with degree } \leq n\right. \\
& \text { for each } \left.x_{i}, i=1, \cdots, s\right\} .
\end{aligned}
$$

Proposition 4.2 (cf. [6], Theorem 1.2.2). Let $w_{i} \in \mathcal{F}\left(C^{2}+\right), i=1,2, \cdots, s$, and let $n \geq 1$ be an integer. Then for all $P \in \mathcal{P}_{2 n-1 ; 5}\left(\mathbb{R}^{s}\right)$, we have

$$
\begin{aligned}
& \int_{\mathbb{R}^{s}} P(X(u)) W^{2}(X(u)) D(X(u)) \\
& =\sum_{k_{s}=1}^{n} \cdots \sum_{k_{1}=1}^{n} \lambda_{k_{1}, n, 1} \cdots \lambda_{k_{s}, n, s} P\left(x_{k_{1}, n, 1} \cdots, x_{k_{s}, n, s}\right),
\end{aligned}
$$

where

$$
\lambda_{k_{i}, n, i}=\int_{-\infty}^{\infty} l_{k_{i}, n, i} w_{i}^{2}\left(x_{i}\right) \mathrm{d} x_{i}, i=1,2, \cdots, s .
$$

Proof. (see [6], pp.12-13). Let $P \in \mathcal{P}_{n-1 ; s}\left(\mathbb{R}^{s}\right)$. From (3.1) and (4.2) we see

$$
\begin{aligned}
P(X) & =L_{n}(P ; X) \\
& =\sum_{k_{s}=1}^{n} \cdots \sum_{k_{1}=1}^{n} l_{k_{1}, n, 1}\left(x_{1}\right) \cdots l_{k_{s}, n, s}\left(x_{s}\right) P\left(x_{k_{1}, n, 1}, \cdots, x_{k_{s}, n, s}\right) .
\end{aligned}
$$

Hence we have

$$
\begin{aligned}
& \int_{\mathbb{R}^{s}} P(X(u)) W^{2}(X(u)) D(X(u)) \\
& =\sum_{k_{s}=1}^{n} \cdots \sum_{k_{1}=1}^{n} \lambda_{k_{1}, n, 1} \cdots \lambda_{k_{s}, n, s} P\left(x_{k_{1}, n, 1} \cdots, x_{k_{s}, n, s}\right),
\end{aligned}
$$

that is, (4.5) holds. Now, we see that (4.5) holds for any $P \in \mathcal{P}_{2 n-1 ; s}\left(\mathbb{R}^{s}\right)$. In fact, for $P \in \mathcal{P}_{2 n-1 ; s}\left(\mathbb{R}^{s}\right)$ we set

$$
\begin{aligned}
& P\left(x_{1}, \cdots, x_{s}\right)=Q\left(x_{1}, \cdots, x_{s}\right) \prod_{i=1}^{s} p_{n, i}\left(x_{i}\right)+R\left(x_{1}, \cdots, x_{s}\right), \\
& Q, R \in \mathcal{P}_{n-1, \cdots, n-1}\left(\mathbb{R}^{s}\right) .
\end{aligned}
$$


Then

$$
\begin{aligned}
& \int_{\mathbb{R}^{s}} P(X(u)) W(X(u)) D(X(u)) \\
& =\int_{\mathbb{R}^{s}} Q(X(u)) \prod_{i=1}^{s} p_{n, j_{i}}\left(u_{j_{i}}\right) W(X(u)) D(X(u)) \\
& +\int_{\mathbb{R}^{s}} R(X(u)) W(X(u)) D(X(u)) \\
& =\int_{\mathbb{R}^{s}} R(X(u)) W(X(u)) D(X(u)) \\
& =\sum_{k_{s}=1}^{n} \cdots \sum_{k_{1}=1}^{n} \lambda_{k_{1}, n, 1} \cdots \lambda_{k_{s}, n, s} R\left(x_{k_{1}, n, 1} \cdots, x_{k_{s}, n, s}\right) \\
& =\sum_{k_{s}=1}^{n} \cdots \sum_{k_{1}=1}^{n} \lambda_{k_{1}, n, 1} \cdots \lambda_{k_{s}, n, s}\left(Q\left(x_{k_{1}, n, 1}, \cdots, x_{k_{s}, n, s}\right) \prod_{i=1}^{s} p_{n, i}\left(x_{k_{i}, n, i}\right)+R\left(x_{k_{1}, n, 1} \cdots, x_{k_{s}, n, s}\right)\right) \\
& =\sum_{k_{s}=1}^{n} \cdots \sum_{k_{1}=1}^{n} \lambda_{k_{1}, n, 1} \cdots \lambda_{k_{s}, n, s} P\left(x_{k_{1}, n, 1}, \cdots, x_{k_{s}, n, s}\right),
\end{aligned}
$$

that is, (4.5) holds for any $P \in \mathcal{P}_{2 n-1 ; s}\left(\mathbb{R}^{s}\right) . \quad \#$

Lemma 4.3 ([7], Theorem 2.1). Let $w=\exp (-Q) \in \mathcal{F}\left(C^{2}+\right), b \in \mathbb{R}$. If $w$ is a Freud-type weight, then we assume $a_{n}=o(1) n^{2 / 3}$. Then there exist constants $C_{1}, C_{2}>0$ such that for every integer $n \geq 1$,

$$
C_{1} \int_{-a_{n}}^{a_{n}}\left(1+x^{2}\right)^{b} \mathrm{~d} x \leq \sum_{j=1}^{n} \lambda_{k, n} w^{-2}\left(x_{k, n}\right)\left(1+x_{k, n}^{2}\right)^{b} \leq C_{2} \int_{-a_{n}}^{a_{n}}\left(1+x^{2}\right)^{b} \mathrm{~d} x .
$$

Proof of Theorem 3.2.

$$
\begin{aligned}
& \sum_{k_{s}=1}^{n} \cdots \sum_{k_{1}=1}^{n} \prod_{i=1}^{s} \lambda_{k_{i}, n, i}\left|f\left(x_{k_{1}, n, 1} \cdots, x_{k_{s}, n, s}\right)\right| \\
& \leq\left\|\prod_{i=1}^{s}\left\{\left(1+x_{i}^{2}\right)^{\beta} w_{i}^{2}\left(x_{i}\right)\right\} f\left(x_{1}, \cdots, x_{s}\right)\right\|_{L^{\infty}\left(\mathbb{R}^{s}\right)} \\
& \quad \cdot \sum_{s=1}^{n} \cdots \sum_{i=1}^{n} \prod_{i=1}^{s}\left\{\lambda_{k_{i}, n, i} w_{i}^{-2}\left(x_{k_{i}, n, i}\right)\left(1+x_{k_{i}, n, i}^{2}\right)^{-\beta}\right\} \\
& \leq\left\|\prod_{i=1}^{s}\left\{\left(1+x_{i}^{2}\right)^{\beta} w_{i}^{2}\left(x_{i}\right)\right\} f\left(x_{1}, \cdots, x_{s}\right)\right\|_{L^{\infty}\left(\mathbb{R}^{s}\right)} \\
& \cdot \int_{-a_{n}^{[s]}}^{a_{n}^{[s]}} \cdots \int_{-a_{n}^{1]}}^{a_{n}^{[1]}} \prod_{i=1}^{s}\left(1+x_{i}^{2}\right)^{-\beta} \mathrm{d} x_{1} \cdots \mathrm{d} x_{s}
\end{aligned}
$$

by Lemma 4.3

$$
\leq C\left\|\prod_{i=1}^{s}\left\{\left(1+x_{i}^{2}\right)^{\beta} w_{i}^{2}\left(x_{i}\right)\right\} f\left(x_{1}, \cdots, x_{s}\right)\right\|_{L^{\infty}\left(\mathbb{R}^{s}\right)} .
$$

Therefore we have (3.4). To prove (3.5) we use (3.4) and Proposition 4.2. For $P \in \mathcal{P}_{n ; s}\left(\mathbb{R}^{s}\right)$ we see 


$$
\begin{aligned}
& \mid \sum_{k_{s}=1}^{n} \cdots \sum_{k_{1}=1}^{n}\left\{\prod_{i=1}^{s} \lambda_{k_{i}, n, i}\right\} f\left(x_{k_{1}, n, 1} \cdots x_{k_{s}, n, s}\right) \\
& -\int_{-\infty}^{\infty} \cdots \int_{-\infty}^{\infty} f\left(x_{1}, \cdots, x_{s}\right) w_{1}^{2}\left(x_{1}\right) \cdots w_{s}^{2}\left(x_{s}\right) \mathrm{d} x_{1} \cdots \mathrm{d} x_{s} \mid \\
& \leq\left|\sum_{k_{s}=1}^{n} \cdots \sum_{k_{1}=1}^{n}\left\{\prod_{i=1}^{s} \lambda_{k_{i}, n, i}\right\}(f-P)\left(x_{k_{1}, n, 1} \cdots x_{k_{s}, n, s}\right)\right| \\
& \quad+\left|\int_{-\infty}^{\infty} \cdots \int_{-\infty}^{\infty}(f-P)\left(x_{1}, \cdots, x_{s}\right) w_{1}^{2}\left(x_{1}\right) \cdots w_{s}^{2}\left(x_{s}\right) \mathrm{d} x_{1} \cdots \mathrm{d} x_{s}\right| \\
& \leq\left|\sum_{k_{s}=1}^{n} \cdots \sum_{k_{1}=1}^{n}\left\{\prod_{i=1}^{s} \lambda_{k_{i}, n, i}\right\}(f-P)\left(x_{k_{1}, n, 1} \cdots x_{k_{s}, n, s}\right)\right| \\
& \quad+\left|\int_{-\infty}^{\infty} \cdots \int_{-\infty}^{\infty}(f-P)\left(x_{1}, \cdots, x_{s}\right) w_{1}^{2}\left(x_{1}\right) \cdots w_{s}^{2}\left(x_{s}\right) \mathrm{d} x_{1} \cdots \mathrm{d} x_{s}\right| \\
& \leq C\left\|\prod_{i=1}^{s}\left\{\left(1+x_{i}^{2}\right)^{\beta} w_{i}^{2}\left(x_{i}\right)\right\}(f-P)\left(x_{1}, \cdots, x_{s}\right)\right\|_{L^{\infty}\left(\mathbb{R}^{s}\right)} \\
& \quad+\left\|\prod_{i=1}^{s}\left\{\left(1+x_{i}^{2}\right)^{\beta} w_{i}^{2}\left(x_{i}\right)\right\}(f-P)\left(x_{1}, \cdots, x_{s}\right)\right\|_{L^{\infty}\left(\mathbb{R}^{s}\right)} \\
& \quad \times \int_{-\infty}^{\infty} \cdots \int_{-\infty}^{\infty} \prod_{i=1}^{s}\left(1+x_{i}^{2}\right)^{-\beta} \mathrm{d} x_{1} \cdots \mathrm{d} x_{s} \\
& \leq C\left\|\prod_{i=1}^{s}\left\{\left(1+x_{i}^{2}\right)^{\beta} w_{i}^{2}\left(x_{i}\right)\right\}(f-P)\left(x_{1}, \cdots, x_{s}\right)\right\|_{L^{\infty}\left(\mathbb{R}^{s}\right)} .
\end{aligned}
$$

Now, we can take $P=P_{n}$ as

$$
\lim _{n \rightarrow \infty}\left\|\prod_{i=1}^{s}\left\{\left(1+x_{i}^{2}\right)^{\beta} w_{i}^{2}\left(x_{i}\right)\right\}\left(f-P_{n}\right)\left(x_{1}, \cdots, x_{s}\right)\right\|_{L^{\infty}\left(\mathbb{R}^{s}\right)}=0 .
$$

In fact, when we put $\prod_{i=1}^{s}\left(1+x_{i}^{2}\right)^{\beta} w_{i}^{2}\left(x_{i}\right) \sim W_{\beta} \in \mathcal{F}\left(C^{2}+\right)$ (see Proposition 2.3), from (3.3) we see

$$
\prod_{i=1}^{s}\left\{T_{i}^{1 / 4}\left(x_{i}\right)\left(1+x_{i}^{2}\right)^{\beta} w_{i}^{2}\right\} f \leq C \prod_{i=1}^{s}\left\{\left(1+x_{i}^{2}\right)^{\beta} w_{i}\left(x_{i}\right)\right\} f \in C_{0}\left(\mathbb{R}^{s}\right) .
$$

Hence from Theorem 3.1 with $W_{\beta}$ we have (4.7). Therefore we conclude (3.5).

Proof of Theorem 3.3. By Proposition 4.2 with $P=L_{n}^{2}(f)$ and Theorem 3.2 with (3.4),

$$
\begin{aligned}
& \left\|\prod_{i=1}^{s} w_{i} L_{n}(f)\right\|_{L^{2}\left(\mathbb{R}^{s}\right)} \\
& =\sum_{k_{s}=1}^{n} \cdots \sum_{k_{1}=1}^{n}\left\{\prod_{i=1}^{s} \lambda_{k_{i}, n, i}\right\}\left\{L_{n}\left(f ; x_{k_{1}, n, 1} \cdots x_{k_{s}, n, s}\right)\right\}^{2} \\
& =\sum_{k_{s}=1}^{n} \cdots \sum_{k_{1}=1}^{n}\left\{\prod_{i=1}^{s} \lambda_{k_{i}, n, i}\right\}\left\{f\left(x_{k_{1}, n, 1} \cdots x_{k_{s}, n, s}\right)\right\}^{2} \\
& \leq C\left\|\prod_{i=1}^{s}\left\{\left(1+x_{i}^{2}\right)^{\beta} w_{i}^{2}\left(x_{i}\right)\right\}\left\{f\left(x_{1}, \cdots, x_{s}\right)\right\}^{2}\right\|_{L^{\infty}\left(\mathbb{R}^{s}\right)} \\
& =C\left\|\prod_{i=1}^{s}\left\{\left(1+x_{i}^{2}\right)^{(\beta / 2)} w_{i}\left(x_{i}\right)\right\} f\left(x_{1}, \cdots, x_{s}\right)\right\|_{L^{\infty}\left(\mathbb{R}^{s}\right)}^{2},
\end{aligned}
$$


that is, we have (3.6). From Theorem 4.1 with $\prod_{i=1}^{s}\left(1+x_{i}^{2}\right)^{\beta / 2} w_{i}^{2}\left(x_{i}\right) \sim W_{\beta / 2} \in \mathcal{F}\left(C^{2}+\right) \quad$ (note Proposition 2.3) and our assumption, there exists $P_{n-1} \in \mathcal{P}_{n-1}$ such that

$$
\lim _{n \rightarrow \infty}\left\|\prod_{i=1}^{s}\left\{\left(1+x_{i}^{2}\right)^{(\beta / 2)} w_{i}\left(x_{i}\right)\right\}\left(f-P_{n-1}\right)\left(x_{1}, \cdots, x_{s}\right)\right\|_{L^{\infty}\left(\mathbb{R}^{s}\right)}^{2}=0 .
$$

Then

$$
\begin{aligned}
& \left\|W\left(f-L_{n}(f)\right)\right\|_{L^{2}\left(\mathbb{R}^{s}\right)} \leq\left\|W\left(f-P_{n-1}\right)\right\|_{L^{2}\left(\mathbb{R}^{s}\right)}+\left\|W L_{n}(f-P)\right\|_{L^{2}\left(\mathbb{R}^{s}\right)} \\
& \leq\left\|\prod_{i=1}^{s}\left\{\left(1+x_{i}^{2}\right)^{(\beta / 2)} w_{i}\left(x_{i}\right)\right\}\left(f-P_{n-1}\right)\left(x_{1}, \cdots, x_{s}\right)\right\|_{L^{\infty}\left(\mathbb{R}^{s}\right)}\left\|\prod_{i=1}^{s}\left(1+x_{i}^{2}\right)^{(-\beta / 2)}\right\|_{L^{2}\left(\mathbb{R}^{s}\right)} \\
& \quad+\left\|\prod_{i=1}^{s}\left\{\left(1+x_{i}^{2}\right)^{(\beta / 2)} w_{i}\left(x_{i}\right)\right\}\left(f-P_{n-1}\right)\left(x_{1}, \cdots, x_{s}\right)\right\|_{L^{\infty}\left(\mathbb{R}^{s}\right)}^{2} \\
& \leq C\left\|\prod_{i=1}^{s}\left\{\left(1+x_{i}^{2}\right)^{(\beta / 2)} w_{i}\left(x_{i}\right)\right\}\left(f-P_{n-1}\right)\left(x_{1}, \cdots, x_{s}\right)\right\|_{L^{\infty}\left(\mathbb{R}^{s}\right)}^{2} \rightarrow 0 \text { as } n \rightarrow \infty .
\end{aligned}
$$

We know the following propositions with respect to one variable.

Proposition 4.4 ([8], Theorem 2.7). Let $w \in \mathcal{F}_{\lambda}\left(C^{3}+\right), 0<\lambda<3 / 2$. Let $1<p \leq 2$ and $\beta>1 / p$. If $f: \mathbb{R}^{s} \rightarrow \mathbb{R}$ is continuous, and satisfies

$$
\left|\left(1+x^{2}\right)^{\beta / 2} T^{1 / 2}(x) \Psi^{-1 / 4}(x) w(x) f(x)\right|<\infty, x \in \mathbb{R},
$$

then we have

$$
\left\|w L_{n}(f)\right\|_{L^{p}(\mathbb{R})} \leq C\left\|\left(1+x^{2}\right)^{\beta / 2} T^{1 / 2}(x) \Psi^{-1 / 4}(x) w f\right\|_{L^{\infty}(\mathbb{R})}, n=1,2, \cdots .
$$

Especially, if

$$
\left|\left(1+x^{2}\right)^{\beta / 2} T^{1 / 2}(x) \Psi^{-1 / 4}(x) w(x) f(x)\right| \in C_{0}(\mathbb{R}),
$$

then we have

$$
\lim _{n \rightarrow \infty}\left\|w\left(f-L_{n}(f)\right)\right\|_{L^{p}(\mathbb{R})}=0 .
$$

Proposition 4.5 ([8], Theorem 2.8). Let $w \in \mathcal{F}_{\lambda}\left(C^{3}+\right), 0<\lambda<3 / 2$, and let satisfy $T_{i}\left(a_{n}^{(i)}\right) \leq C n^{1 / 2}$. Let $2<p \leq \infty$ and $\beta>1 / p$. Furthermore we assume

$$
Q\left(\frac{a_{n}}{4}\right) \geq C\left(\log \left(Q\left(a_{n}\right)\right)\right)^{4} .
$$

If $f: \mathbb{R} \rightarrow \mathbb{R}$ satisfies

$$
\left|\left(1+x^{2}\right)^{\beta / 2} T^{1 / 2}(x) \Psi^{-1 / 4}(x) w(x) f(x)\right| \leq C, x \in \mathbb{R},
$$

then we have

$$
\begin{aligned}
& \left\|\Psi^{3 / 4}(x) w L_{n}(f)\right\|_{L^{p}(\mathbb{R})} \leq C\left\|\left(1+x^{2}\right)^{\beta / 2} T^{1 / 2}(x) \Psi^{-1 / 4}(x) w f\right\|_{L^{\infty}(\mathbb{R})}, \\
& n=1,2, \cdots .
\end{aligned}
$$


Especially, if

$$
\left|\left(1+x^{2}\right)^{\beta / 2} T^{1 / 2}(x) \Psi^{-1 / 4}(x) w(x) f(x)\right| \in C_{0}(\mathbb{R}),
$$

we have

$$
\lim _{n \rightarrow \infty}\left\|w\left(f-L_{n}(f)\right)\right\|_{L^{p}(\mathbb{R})}=0 .
$$

Proof of Theorem 3.4. We use Proposition 4.4 (4.8).

$$
\begin{aligned}
& \left\|W L_{n}(f)\right\|_{L^{p}\left(\mathbb{R}^{s}\right)}^{p} \\
& =\int_{\mathbb{R}_{2 \leq}^{s-1}} \sum_{k_{s}=1}^{n} \cdots \sum_{k_{2}=1}^{n}\left\{\prod_{i=2}^{s} w_{i}\left(x_{i}\right)\right\}^{p} \int_{\mathbb{R}_{(1)}}\left|w_{1}\left(x_{1}\right) \sum_{k_{1}=1}^{n} f\left(x_{k_{1}, n, 1}, \cdots, x_{k_{s}, n, s}\right) l_{k_{1}, n, 1}\left(x_{1}\right)\right|^{p} \mathrm{~d} x_{1} \\
& \times\left|\prod_{i=2}^{s} l_{k_{i}, n, i}\left(x_{i}\right)\right|^{p} \mathrm{~d} x_{2} \cdots \mathrm{d} x_{s} \\
& \leq C_{1} \int_{\mathbb{R}_{2 \leq}^{s-1}} \sum_{k_{s}=1}^{n} \cdots \sum_{k_{2}=1}^{n}\left\{\prod_{i=2}^{s} w_{i}\left(x_{i}\right)\right\}^{p} \\
& \times\left\|\left(1+x_{1}^{2}\right)^{\beta / 2} T_{1}^{1 / 2}\left(x_{1}\right) \Psi_{1}^{-1 / 4}\left(x_{1}\right) w_{1}\left(x_{1}\right) f\left(x_{1}, x_{2}, \cdots, x_{s}\right)\right\|_{L^{\infty}\left(\mathbb{R}_{(1)}\right)}^{p} \\
& \times\left\{\prod_{i=2}^{s} l_{k_{i}, n, i}\left(x_{i}\right)\right\} \mathrm{d} x_{2} \cdots \mathrm{d} x_{s} \\
& =C_{1} \|\left\{\left(1+x_{1}^{2}\right)^{\beta / 2} T_{1}^{1 / 2}\left(x_{1}\right) \Psi_{1}^{-1 / 4}\left(x_{1}\right) w_{1}\left(x_{1}\right)\right\}^{p} \\
& \times \int_{\mathbb{R}_{2 \leq}^{s-1}} \sum_{k_{s}=1}^{n} \cdots \sum_{k_{2}=1}^{n}\left\{\prod_{i=2}^{s} w_{i}\left(x_{i}\right)\right\}^{p}\left|f\left(x_{1}, x_{2}, \cdots, x_{s}\right)\right|^{p} \\
& \times\left\{\prod_{i=2}^{s} l_{k_{i}, n, i}\left(x_{i}\right)\right\} \mathrm{d} x_{2} \cdots \mathrm{d} x_{s} \|_{L^{\infty}\left(\mathbb{R}_{(1)}\right)} \\
& \leq C_{2} \|\left\{\left(1+x_{1}^{2}\right)^{\beta / 2} T_{1}^{1 / 2}\left(x_{1}\right) \Psi_{1}^{-1 / 4}\left(x_{1}\right) w_{1}\left(x_{1}\right)\left(1+x_{2}^{2}\right)^{\beta / 2} T_{2}^{1 / 2}\left(x_{1}\right) \Psi_{2}^{-1 / 4}\left(x_{2}\right) w_{2}\left(x_{2}\right)\right\}^{p} \\
& \times \int_{\mathbb{R}_{35}^{s-1}} \sum_{k_{s}=1}^{n} \cdots \sum_{k_{3}=1}^{n}\left\{\prod_{i=3}^{s} w_{i}\left(x_{i}\right)\right\}^{p}\left|f\left(x_{1}, x_{2}, \cdots, x_{s}\right)\right|^{p} \\
& \times\left\{\prod_{i=3}^{s} l_{k_{i}, n, i}\left(x_{i}\right)\right\} \mathrm{d} x_{3} \cdots \mathrm{d} x_{s} \|_{L^{\infty}\left(\mathbb{R}_{\leq 2}\right)}^{p} \\
& \leq \cdots \\
& \leq C_{s}\left\|\prod_{i=1}^{s}\left\{\left(1+x_{i}^{2}\right)^{\beta / 2} T_{i}^{1 / 2}\left(x_{1}\right) \Psi_{i}^{-1 / 4}\left(x_{i}\right) w_{i}\left(x_{i}\right)\right\} f\left(x_{1}, x_{2}, \cdots, x_{s}\right)\right\|_{L^{\infty}\left(\mathbb{R}^{s}\right)}^{p} .
\end{aligned}
$$

Hence we have (3.9).

Next we show (3.10). There exists $P_{n} \in \mathcal{P}_{n ; s}\left(\mathbb{R}^{s}\right)$ such that

$$
\begin{aligned}
& \left\|\prod_{i=1}^{s}\left\{\left(1+x_{i}^{2}\right)^{\beta / 2} T_{i}^{1 / 2}\left(x_{i}\right) \Psi_{i}^{-1 / 4}\left(x_{i}\right)\right\} W\left(f-P_{n}\right)\right\|_{L^{\infty}\left(\mathbb{R}^{s}\right)} \\
& \leq C E_{\infty, n ; s}\left(\prod_{i=1}^{s}\left\{\left(1+x_{i}^{2}\right)^{\beta / 2} T_{i}^{1 / 2}\left(x_{i}\right) \Psi_{i}^{-1 / 4}\left(x_{i}\right)\right\} W, f\right) \\
& \left\|\prod_{i=1}^{s}\left\{\left(1+x_{i}^{2}\right)^{\beta / 2} T_{i}^{1 / 2}\left(x_{i}\right) \Psi_{i}^{-1 / 4}\left(x_{i}\right)\right\} W\left(f-P_{n}\right)\right\|_{L^{\infty}\left(\mathbb{R}^{s}\right)} \\
& \leq C E_{\infty, n ; s}\left(\prod_{i=1}^{s}\left\{\left(1+x_{i}^{2}\right)^{\beta / 2} T_{i}^{1 / 2}\left(x_{i}\right) \Psi_{i}^{-1 / 4}\left(x_{i}\right)\right\} W, f\right),
\end{aligned}
$$


where $P_{n} \in \mathcal{P}_{n ; s}$. Then

$$
\begin{aligned}
& \left\|W\left(f-L_{n}(f)\right)\right\|_{L^{p}\left(\mathbb{R}^{s}\right)} \leq\left\|W\left(f-P_{n}\right)\right\|_{L^{p}\left(\mathbb{R}^{s}\right)}+\left\|W L_{n}\left(f-P_{n}\right)\right\|_{L^{p}\left(\mathbb{R}^{s}\right)} \\
& \leq\left\|W\left(f-P_{n}\right)\right\|_{L^{p}\left(\mathbb{R}^{s}\right)}+C\left\|\prod_{i=1}^{s}\left\{\left(1+x_{i}^{2}\right)^{\beta / 2} T_{i}^{1 / 2}\left(x_{i}\right) \Psi_{i}^{-1 / 4}\left(x_{i}\right)\right\} W\left(f-P_{n}\right)\right\|_{L^{\infty}\left(\mathbb{R}^{s}\right)} \\
& \leq\left\|\prod_{i=1}^{s}\left\{\left(1+x_{i}^{2}\right)^{\beta / 2} T_{i}^{1 / 2}\left(x_{i}\right) \Psi_{i}^{-1 / 4}\left(x_{i}\right)\right\} W\left(f-P_{n}\right)\right\|_{L^{\infty}\left(\mathbb{R}^{s}\right)} \\
& \quad \times\left\|\prod_{i=1}^{s}\left\{\left(1+x_{i}^{2}\right)^{-\beta / 2} T_{i}^{-1 / 2}\left(x_{i}\right) \Psi_{i}^{1 / 4}\left(x_{i}\right)\right\}\right\|_{L^{p}\left(\mathbb{R}^{s}\right)} \\
& \quad+C\left\|\prod_{i=1}^{s}\left\{\left(1+x_{i}^{2}\right)^{\beta / 2} T_{i}^{1 / 2}\left(x_{i}\right) \Psi_{i}^{-1 / 4}\left(x_{i}\right)\right\} W\left(f-P_{n}\right)\right\|_{L^{\infty}\left(\mathbb{R}^{s}\right)} \\
& \leq C E_{\infty, n ; s}\left(\prod_{i=1}^{s}\left\{\left(1+x_{i}^{2}\right)^{\beta / 2} T_{i}^{1 / 2}\left(x_{i}\right) \Psi_{i}^{-1 / 4}\left(x_{i}\right)\right\} W, f\right) \rightarrow 0 \text { as } n \rightarrow 0 .
\end{aligned}
$$

The last convergence follows from (3.9) and Theorem 3.1 with $\prod_{i=1}^{s}\left\{\left(1+x_{i}^{2}\right)^{\beta / 2} T_{i}^{1 / 2}\left(x_{i}\right) \Psi_{i}^{-1 / 4}\left(x_{i}\right)\right\} \sim W^{*} \in \mathcal{F}\left(C^{2}+\right) \quad$ (note Proposition 2.3). Consequently, we have (3.11).

Proof of Theorem 3.5. As the proof of Theorem 3.4 we can show Theorem 3.5 by Proposition 4.5 (4.9). Then we also note Remark 3.6 (1) and (3). \#

\section{Proofs of Theorems 3.7, 3.8 and 3.9}

In this section, we characterize the best approximation polynomial (cf. [5]).

Proof of Theorem 3.7. We consider the polynomial class

$$
\mathcal{T}=\left\{P\left(x_{1}, \cdots, x_{s}\right)=\sum_{k_{i}=0,1 \leq i \leq s}^{n} a_{k_{1}, \cdots, k_{s}} x_{1}^{k_{1}} \cdots x_{s}^{k_{s}} ;\|(f-P) W\|_{L^{p}\left(\mathbb{R}^{s}\right)} \leq\|f W\|_{L^{p}\left(\mathbb{R}^{s}\right)}\right\} .
$$

Since

$$
\|(f-0) W\|_{L^{p}\left(\mathbb{R}^{s}\right)}=\|f W\|_{L^{p}\left(\mathbb{R}^{s}\right)},
$$

the set $\mathcal{T}$ is not empty. Now we select the sequence

$$
\begin{aligned}
& \left\{P_{m, n}\left(x_{1}, \cdots, x_{s}\right)=\sum_{k_{i}=0,1 \leq i \leq s}^{n} a_{k_{1}, \cdots, k_{s} ; m} x_{1}^{k_{1}} \cdots x_{s}^{k_{s}}\right\}_{m=0}^{\infty} \text { such that } \\
& \inf _{a_{k_{1}, \cdots, k_{s} ; m}}\left\|\left(f-P_{m, n}\right) W\right\|_{L^{p}\left(\mathbb{R}^{s}\right)}=E_{p, n ; s}(W ; f) .
\end{aligned}
$$

Here we see that $\left|a_{m}\right|:=\max _{k_{j}=0,1, \cdots, n ; 1<j \leq s}\left|a_{k_{1}, \cdots, k_{s} ; m}\right|$ is bounded. In fact, if it is unbounded, then for

$$
Q_{m, n}\left(x_{1}, \cdots, x_{s}\right)=P_{m, n}\left(x_{1}, \cdots, x_{s}\right) / a_{m}=\sum_{k_{i}=0,1 \leq i \leq s}^{n} b_{k_{1}, \cdots, k_{s} ; m} x_{1}^{k_{1}} \cdots x_{s}^{k_{s}},
$$

we see $\left|b_{k_{1}, \cdots, k_{s} ; m}\right| \leq 1$. Then we can take a subsequence $\left\{m_{l}\right\}_{l=1}^{\infty}$ and a fixed term $x_{1}^{k_{1 ; 0}} \cdots x_{s}^{k_{s ; 0}}$ such that

$$
Q_{m_{l}, n}\left(x_{1}, \cdots, x_{s}\right)=x_{1}^{k_{1,0}} \cdots x_{s}^{k_{s, 0}}+\sum_{k_{i}=0, k_{i} \neq k_{i, 0}, 1 \leq i \leq s}^{n} b_{k_{1}, \cdots, k_{s} ; m_{l}} x_{1}^{k_{1}} \cdots x_{s}^{k_{s}} .
$$


We can suppose $b_{k_{1}, \cdots, k_{s} ; m_{l}} \rightarrow b_{k_{1}, \cdots, k_{s}}$ as $l \rightarrow \infty$ (if we need it, then we consider a subsequence). Now, we see that there exists $M>0$ such that $\left\|P_{m_{l}} W\right\|_{L^{\infty}\left(\mathbb{R}^{s}\right)}<M$, so we have

$$
\left\|Q_{m_{l}, n} W\right\|_{L^{\infty}\left(\mathbb{R}^{s}\right)} \rightarrow 0
$$

that is,

$$
Q_{n}\left(x_{1}, \cdots, x_{s}\right):=x_{1}^{k_{1} ; 0} \cdots x_{s}^{k_{s ; 0}}+\sum_{k_{i}=0, k_{i} \neq k_{i, 0}, 1 \leq i \leq s}^{n} b_{k_{1}, \cdots, k_{s}} x_{1}^{k_{1}} \cdots x_{s}^{k_{s}}=0 .
$$

This is impossible because the $\left\{x_{1}^{k_{1}} \cdots x_{s}^{k_{s}}\right\}$ are linear independent. Hence $\left|a_{m}\right|:=\max _{k_{j}=0,1, \cdots, n ; 1<j \leq s}\left|a_{k_{1}, \cdots, k_{s} ; m}\right|$ is bounded. Now we repeat the method as above. If we select the sequence $a_{k_{1}, \cdots, k_{s} ; m} \rightarrow a_{k_{1}, \cdots, k_{s}}$ as $l \rightarrow \infty$ (if we need it, then we consider a subsequence), then we have

$$
\left\|\left(f-\sum_{k_{i}=0,1 \leq i \leq s}^{n} a_{k_{1}, \cdots, k_{s}} x_{1}^{k_{1}} \cdots x_{s}^{k_{s}}\right) W\right\|_{L^{p}(\mathbb{R})}=E_{p, n ; s}(W ; f) .
$$

Then we put $P_{n ; f}:=\sum_{k_{i}=0,1 \leq i \leq s}^{n} a_{k_{1}, \cdots, k_{s}} x_{1}^{k_{1}} \cdots x_{s}^{k_{s}}$.

\#

Proof of Theorem 3.8. Let

$$
\left\|W\left(f-P_{n ; f}\right)\right\|_{L^{\infty}\left(\mathbb{R}^{s}\right)}=E_{n ; s}(W, f),
$$

where $P_{n ; f} \in \mathcal{P}_{n ; s}\left(\mathbb{R}^{s}\right)$. We see that the theorem is trivial if $E_{n ; s}(W, f)=0$. So we may assume $E_{n ; s}(W, f)>0$. If (3.14) is not true, there exists a polynomial $Q \in \mathcal{P}_{n ; s}$ such that

$$
\max _{\left(x_{1}, \cdots, x_{s}\right) \in A}\left[W\left(x_{1}, \cdots, x_{s}\right)\left(f\left(x_{1}, \cdots, x_{s}\right)-P_{n ; f}\left(x_{1}, \cdots, x_{s}\right)\right)\right] Q\left(x_{1}, \cdots, x_{s}\right)=-2 \varepsilon
$$

for some $\varepsilon>0$. By the continuity of the function, there exists an open subset $G ; A \subset G$, such that

$$
W\left(x_{1}, \cdots, x_{s}\right)\left[f\left(x_{1}, \cdots, x_{s}\right)-P_{n, f}\left(x_{1}, \cdots, x_{s}\right)\right] Q\left(x_{1}, \cdots, x_{s}\right)<-\varepsilon,\left(x_{1}, \cdots, x_{s}\right) \in G .
$$

For $\lambda>0$ small enough we put $R=P_{n ; f}-\lambda Q$, and let

$$
M=\sup _{\left(x_{1}, \cdots, x_{s}\right) \in \mathbb{R}^{s}} W\left(x_{1}, \cdots, x_{s}\right)\left|Q\left(x_{1}, \cdots, x_{s}\right)\right| .
$$

First, for $\left(x_{1}, \cdots, x_{s}\right) \in G$ we see

$$
\begin{aligned}
\mid & \left.W\left(x_{1}, \cdots, x_{s}\right)\left(f\left(x_{1}, \cdots, x_{s}\right)-R\left(x_{1}, \cdots, x_{s}\right)\right)\right|^{2} \\
= & \left|W\left(x_{1}, \cdots, x_{s}\right)\left(f\left(x_{1}, \cdots, x_{s}\right)-P_{n ;} f\left(x_{1}, \cdots, x_{s}\right)+\lambda Q\left(x_{1}, \cdots, x_{s}\right)\right)\right|^{2} \\
= & \left|W\left(x_{1}, \cdots, x_{s}\right)\left(f\left(x_{1}, \cdots, x_{s}\right)-P_{n ; f}\left(x_{1}, \cdots, x_{s}\right)\right)\right|^{2} \\
& +2 \lambda\left[W\left(x_{1}, \cdots, x_{s}\right)\left(f\left(x_{1}, \cdots, x_{s}\right)-P_{n ; f}\left(x_{1}, \cdots, x_{s}\right)\right)\right] Q\left(x_{1}, \cdots, x_{s}\right) \\
& +\lambda^{2} W^{2}\left(x_{1}, \cdots, x_{s}\right)\left|Q\left(x_{1}, \cdots, x_{s}\right)\right|^{2} \\
< & E_{n ; s}(W, f)^{2}-2 \lambda \varepsilon+\lambda^{2} M^{2} .
\end{aligned}
$$


If we take $\lambda<M^{-2} \varepsilon$, then we obtain

$$
\begin{aligned}
& \left|W\left(x_{1}, \cdots, x_{s}\right)\left(f\left(x_{1}, \cdots, x_{s}\right)-R\left(x_{1}, \cdots, x_{s}\right)\right)\right|^{2} \\
& <E_{n ; s}(W, f)^{2}-2 \lambda \varepsilon+\lambda \varepsilon=E_{n ; s}(W, f)^{2}-\lambda \varepsilon,\left(x_{1}, \cdots, x_{s}\right) \in G .
\end{aligned}
$$

Next, we assume $\left(x_{1}, \cdots, x_{s}\right) \in G^{c}$ (the complement of $G$ ). For large enough $K_{1}, K_{2}>0$ there exists $\delta_{1}>0$ such that

$$
W\left(x_{1}, \cdots, x_{s}\right)\left|f\left(x_{1}, \cdots, x_{s}\right)\right|<\frac{E_{n ; s}(W, f)}{2}-\delta_{1},\left|\left(x_{1}, \cdots, x_{s}\right)\right| \geq K_{1},
$$

and

$$
W\left(x_{1}, \cdots, x_{s}\right)\left|P_{n ; f}\left(x_{1}, \cdots, x_{s}\right)\right|<\frac{E_{n ; s}(W, f)}{2}-\delta_{1},\left|\left(x_{1}, \cdots, x_{s}\right)\right| \geq K_{2},
$$

that is,

$$
\begin{aligned}
& \left|W\left(x_{1}, \cdots, x_{s}\right)\left(f\left(x_{1}, \cdots, x_{s}\right)-P_{n ; f}\left(x_{1}, \cdots, x_{s}\right)\right)\right| \\
& <E_{n ; s}(W, f)-2 \delta_{1}, \quad\left|\left(x_{1}, \cdots, x_{s}\right)\right| \geq \max \left\{K_{1}, K_{2}\right\} .
\end{aligned}
$$

Then we also see that there exists $\delta_{2}>0$ such that

$$
\begin{aligned}
& \left|W\left(x_{1}, \cdots, x_{s}\right)\left(f\left(x_{1}, \cdots, x_{s}\right)-P_{n ; f}\left(x_{1}, \cdots, x_{s}\right)\right)\right| \\
& <E_{n ; s}(W, f)-\delta_{2},\left(x_{1}, \cdots, x_{s}\right) \in G^{c},\left|\left(x_{1}, \cdots, x_{s}\right)\right| \leq \max \left\{K_{1}, K_{2}\right\} .
\end{aligned}
$$

Let $\delta:=\min \left\{2 \delta_{1}, \delta_{2}\right\}>0$, and let $\left(x_{1}, \cdots, x_{s}\right) \in G^{c}$. Then, if we take $\lambda>0$ so small that $\lambda<(2 M)^{-1} \delta$, we see

$$
\begin{aligned}
& \left|W\left(x_{1}, \cdots, x_{s}\right)\left(f\left(x_{1}, \cdots, x_{s}\right)-R\left(x_{1}, \cdots, x_{s}\right)\right)\right| \\
& \leq\left|W\left(x_{1}, \cdots, x_{s}\right)\left(f\left(x_{1}, \cdots, x_{s}\right)-P_{n ; f}\left(x_{1}, \cdots, x_{s}\right)\right)\right| \\
& +\lambda W\left(x_{1}, \cdots, x_{s}\right)\left|Q\left(x_{1}, \cdots, x_{s}\right)\right| \\
& \leq E_{n ; s}(W, f)-\delta+\frac{\delta}{2}=E_{n ; s}(W, f)-\frac{\delta}{2} .
\end{aligned}
$$

From (5.1) and (5.2) we see that the condition (3.14) is necessary.

Next we will show that (3.14) is also sufficient. Let $R \in \mathcal{P}_{n ; s}$ be arbitrary polynomial. Then there exists a point $\left(x_{1,0}, \cdots, x_{s, 0}\right) \in A$ such that for $Q=P-R$,

$$
\left[W\left(x_{1}, \cdots, x_{s}\right)\left(f\left(x_{1,0}, \cdots, x_{s, 0}\right)-P\left(x_{1,0}, \cdots, x_{s, 0}\right)\right)\right] Q\left(x_{1,0}, \cdots, x_{s, 0}\right) \geq 0 .
$$

Then we see

$$
\begin{aligned}
& \left|W\left(x_{1,0}, \cdots, x_{s, 0}\right)\left(f\left(x_{1,0}, \cdots, x_{s, 0}\right)-R\left(x_{1,0}, \cdots, x_{s, 0}\right)\right)\right|^{2} \\
& =\left|W\left(x_{1,0}, \cdots, x_{s, 0}\right)\left(f\left(x_{1,0}, \cdots, x_{s, 0}\right)-P\left(x_{1,0}, \cdots, x_{s, 0}\right)\right)\right|^{2} \\
& \quad+2\left[W\left(x_{1,0}, \cdots, x_{s, 0}\right)\left(f\left(x_{1,0}, \cdots, x_{s, 0}\right)-P\left(x_{1,0}, \cdots, x_{s, 0}\right)\right)\right] Q\left(x_{1,0}, \cdots, x_{s, 0}\right) \\
& \quad+\left|W\left(x_{1,0}, \cdots, x_{s, 0}\right) Q\left(x_{1,0}, \cdots, x_{s, 0}\right)\right|^{2} \\
& \geq\left|W\left(x_{1,0}, \cdots, x_{s, 0}\right)\left(f\left(x_{1,0}, \cdots, x_{s, 0}\right)-P\left(x_{1,0}, \cdots, x_{s, 0}\right)\right)\right|^{2}=\mid W(f-P) \|_{L^{\infty}(\mathbb{R})}^{2} .
\end{aligned}
$$


This means that there is not $R$ with $\|W(f-R)\|_{L^{\infty}(\mathbb{R})}<\|W(f-P)\|_{L^{\infty}(\mathbb{R})}$, that is, $P$ is the best of approximation polynomial.

Proof of 3.9. Let the condition (3.17) be satisfied. We see

$$
\begin{aligned}
& \int_{\mathbb{R}^{s}}|\{f(X)-P(X)\} W(X)|^{p} D(X) \\
& =\int_{\mathbb{R}^{s}}\{f(X)-P(X)\}|f(X)-P(X)|^{p-1} \operatorname{sign}\{f(X)-P(X)\} W^{p}(X) D(X) \\
& =\int_{\mathbb{R}^{s}}\{f(X)-Q(X)\}|f(X)-P(X)|^{p-1} \operatorname{sign}\{f(X)-P(X)\} W^{p}(X) D(X) \\
& \leq \int_{\mathbb{R}^{s}}|f(X)-Q(X)||f(X)-P(X)|^{p-1} W^{p}(X) D(X) \\
& \leq\left[\int_{\mathbb{R}^{s}}|\{f(X)-Q(X)\} W(X)|^{p} D(X)\right]^{\frac{1}{p}}\left[\int_{\mathbb{R}^{s}}|\{f(X)-P(X)\} W|^{p} D(X)\right]^{1-\frac{1}{p}},
\end{aligned}
$$

that is,

$$
\left[\int_{\mathbb{R}^{s}}\left\{\left.\{f(X)-P(X)\} W(X)\right|^{p} D(X)\right]^{\frac{1}{p}} \leq\left[\int_{\mathbb{R}^{s}}|\{f(X)-Q(X)\} W(X)|^{p} D(X)\right]^{\frac{1}{p}} .\right.
$$

Hence $P(X)$ is the best approximation polynomial.

Next we give the converse assertion. We suppose (3.17). However if $p=1$, we also assume that $f(X)-P(X)$ vanish only on a set of measure zero. (3.17) is equivalent to

$$
\int_{\mathbb{R}^{s}} \phi_{K}(X)|f(X)-Q(X)|^{p-1}[\operatorname{sign}(f(X)-Q(X))] W^{p}(X) D(X)=0
$$

for all $\phi_{K}, K=\left(k_{1}, \cdots, k_{s}\right)\left(0 \leq k_{j} \leq n\right)$. Now we assume that for some $K$,

$$
\int_{\mathbb{R}^{s}} \phi_{K}(X)|f(X)-Q(X)|^{p-1}[\operatorname{sign}(f(X)-Q(X))] W^{p}(X) D(X)=\delta \neq 0,
$$

then it would be possible to find $\lambda$ so small on the basis of absolute magnitude that

$$
\begin{aligned}
& \lambda \int_{\mathbb{R}^{s}} \phi_{K}(X)\left|f(X)-Q(X)-\lambda \phi_{K}(X)\right|^{p-1} \\
& \times\left[\operatorname{sign}(f(X)-Q(X))-\lambda \phi_{K}(X)\right] W^{p}(X) D(X)>0 .
\end{aligned}
$$

But then

$$
\begin{aligned}
& \int_{\mathbb{R}^{s}}\left|\left\{f(X)-P(X)-\lambda \phi_{K}(X)\right\} W(X)\right|^{p} D(X) \\
& =\int_{\mathbb{R}^{s}}\left\{f(X)-P(X)-\lambda \phi_{K}(X)\right\}\left|f(X)-P(X)-\lambda \phi_{K}(X)\right|^{p-1} \\
& \quad \times \operatorname{sign}\{f(X)-P(X)\} W^{p}(X) D(X) \\
& =\int_{\mathbb{R}^{s}}\{f(X)-P(X)\}\left|f(X)-P(X)-\lambda \phi_{K}(X)\right|^{p-1} \\
& \quad \times \operatorname{sign}\left\{f(X)-P(X)-\lambda \phi_{K}(X)\right\} W^{p}(X) D(X) \\
& \quad-\lambda \int_{\mathbb{R}^{s}} \phi_{K}(X)\left|f(X)-P(X)-\lambda \phi_{K}(X)\right|^{p-1} \\
& \quad \times \operatorname{sign}\left\{f(X)-P(X)-\lambda \phi_{K}(X)\right\} W^{p}(X) D(X) \\
& <\int_{\mathbb{R}^{s}}|f(X)-P(X)|\left|f(X)-P(X)-\lambda \phi_{K}(X)\right|^{p-1} W^{p}(X) D(X) \\
& \leq\left[\int_{\mathbb{R}^{s}}|\{f(X)-P(X)\} W(X)|^{p} D(X)\right]^{\frac{1}{p}} \\
& \quad \times\left[\int_{\mathbb{R}^{s}}\left|\left\{f(X)-P(X)-\lambda \phi_{K}(X)\right\} W(X)\right|^{p} D(X)\right]^{1-\frac{1}{p}} .
\end{aligned}
$$


Consequently,

$$
\begin{aligned}
& {\left[\int_{\mathbb{R}^{s}}\left|\left\{f(X)-P(X)-\lambda \phi_{K}(X)\right\} W(X)\right|^{p} D(X)\right]^{\frac{1}{p}}} \\
& <\left[\int_{\mathbb{R}^{s}}|\{f(X)-P(X)\} W(X)|^{p} D(X)\right]^{\frac{1}{p}},
\end{aligned}
$$

and we arrive at a contradiction on the assumption concerning the polynomial $P(X)$. \#

\section{References}

[1] Jung, H.S. and Sakai, R. (2009) Specific Examples of Exponential Weights. Communications of the Korean Mathematical Society, 24, 303-319. https://doi.org/10.4134/CKMS.2009.24.2.303

[2] Levin, A.L. and Lubinsky, D.S. (2001) Orthogonal Polynomials for Exponential Weights. Springer, New York. https://doi.org/10.1007/978-1-4613-0201-8

[3] Sakai, R. and Suzuki, N. (2013) Mollification of Exponential Weights and Its Application to the Markov-Bernstein Inequality. The Pioneer Journal of Mathematics, 7 , 83-101.

[4] Sakai, R. A Study of Weighted Polynomial Approximations with Several Variables (I). Applied Mathematics. (Unpublished)

[5] Timan, A.F. (1963) Theory of Approximation of Functions of a Real Variable. Pergamon Press, Oxford.

[6] Mhaskar, H.N. (1996) Introduction to the Theory of Weighted Polynomial Approximation. World Scientific, Singapore.

[7] Sakai, R. (2015) Quadrature Formula with Exponential-Type Weights. Pioneer Journal of Mathematics and Mathematical Sciences, 14, 1-23.

[8] Jung, H.S. and Sakai, R. (2016) $L_{p}$-Convergence of Lagrange Interpolation Polynomials with Regular Symmetric Exponential Type Weight. Global Journal of Pure and Applied Mathematics, 12, 797-822. 
Submit or recommend next manuscript to SCIRP and we will provide best service for you:

Accepting pre-submission inquiries through Email, Facebook, LinkedIn, Twitter, etc. A wide selection of journals (inclusive of 9 subjects, more than 200 journals)

Providing 24-hour high-quality service

User-friendly online submission system

Fair and swift peer-review system

Efficient typesetting and proofreading procedure

Display of the result of downloads and visits, as well as the number of cited articles Maximum dissemination of your research work

Submit your manuscript at: http://papersubmission.scirp.org/

Or contact am@scirp.org 